\title{
A FURTHER GENERALIZATION \\ OF MIDPOINT CONVEXITY OF MULTIMAPS \\ TOWARDS COMMON FIXED POINT THEOREMS AND APPLICATIONS
}

\author{
Irene Benedetti - Anna Martellotti
}

\begin{abstract}
We furtherly generalize midpoint convexity for multivalued maps and derive Fixed Point Theorems and Common Fixed Point Theorems without requiring strong compactness. As an application we obtain some Best Approximation results, and minimax and variational inequalities.
\end{abstract}

\section{Introduction and preliminaries}

Fixed point Theorems for multimaps without compactness have always been object of mathematical investigation.

In particular in the literature one finds Fixed Point Theorems for maps, multimaps and for families of multimaps in Banach spaces replacing norm compactness with weak compactness (see, e.g. [3], [6], [10], [18]).

In the present paper we shall develop the investigation, started in [8], relative to fixed points for multimaps defined on a weakly compact subset $K$ of a Banach space $X$, but with regularity assumptions only relative to the strong topology. In this setting self multimaps may fail to have a fixed point, unless some further conditions are assumed.

2010 Mathematics Subject Classification. Primary: 47H10; Secondary: 47H04.

Key words and phrases. Common fixed points, midpoint convexity, commuting families of multimaps, best approximations, variational inequalities. 
In [17] Nikodem proposed a definition of convexity for multimaps. A multimap $F: K \multimap X$ is said to be convex if for any $x, y \in K$

$$
\lambda F(x)+(1-\lambda) F(y) \subseteq F(\lambda x+(1-\lambda) y) .
$$

Next in $[8]$ the concept has been generalized in the case of single valued functions, and then in [5] furtherly extended to the case of multimaps, introducing the concept of strong and weak midpoint linearity. A multimap $F: K \multimap X$ is said to be strongly midpoint linear if for any $r>0$ the set

$$
F_{r}=\left\{x \in K: x \in F(x)+r X_{1}\right\}
$$

is convex, weakly midpoint linear if for any weak neighborhood of $0, V \in \mathcal{B}$ the set

$$
F_{V}=\{x \in K: x \in F(x)+\bar{V}\}
$$

is convex.

Again in [5] we proposed a weaker form of midpoint linearity, labelled as piecewise midpoint linearity as follows: $F$ is said to be piecewise strongly midpoint linear if for any $r>0$ there exists $s(r) \in] 0, r]$ and a finite decomposition of $K$, say $\mathcal{D}_{s}=\left\{D_{1}, \ldots, D_{n}\right\}$, such that the sets $F_{s(r)} \cap D_{i}$ are convex for any $i=1, \ldots, n$, piecewise weakly midpoint linear provided for any $U \in \mathcal{B}$ there exists $V \in \mathcal{B}, V \subseteq U$ and a finite decomposition of $K$, say $\mathcal{D}_{V}=\left\{D_{1}, \ldots, D_{n}\right\}$, such that the sets $F_{V(U)} \cap D_{i}$ are convex for any $i=1, \ldots, n$.

Indeed, piecewise midpoint linearity in both senses ensures that the sets $F_{r}$ respectively $F_{V}$ are weakly closed, thus allowing the use of the Finite Intersection Property (F.I.P.) in the weakly compact domain $K$, in order to achieve Fixed Point Theorems.

In this paper we furtherly generalize the piecewise midpoint linearities proposed in [5], by assuming a property that we named nicety; this assumption is strictly more general than the weak upper semicontinuity, but it proves to be sufficient to obtain the weak closure of the set of fixed points $P(F)$. Hence we can prove a Fixed Point Theorem for a nice self multimap which is upper semicontinuous when $K$ is equipped with the strong topology (see Theorems 2.7 and 2.8 below).

The weak closure of the set $P(F)$ in a weakly compact domain immediately suggested the investigation of common fixed points. We faced the problem in two frameworks: common fixed points for a pair of multimaps and for a larger family of multimaps. This is the object of Section 2. In particular we prove the suitable versions of some Common Fixed Point Theorems appearing in the literature. The result in [16] suggested a Common Fixed Point Theorem based upon commuting families of multimaps, and its several generalizations, as for instance in [7], [1], while two results without commutativity are inspired by analogous settings in Balaj [3] and in $\mathrm{Hu}-\mathrm{Papageorgiou} \mathrm{[13].}$ 
The final part of the paper is devoted to applications: in Section 3.1 Common Fixed Point Theorems for a pair of multimaps are applied to the theory of Best Approximations as found in the literature (compare to, e.g. [7], [21], [15]). Finally Common Fixed Point Theorems for families of multimaps find their applications to minimax and variational inequalities of Park's [19], [20] type in Section 3.2.

In the whole paper $(X,\|\cdot\|)$ is a Banach space, $X^{*}$ its dual space. $X_{w}$ denotes the space $X$ endowed with the weak topology; $X_{1}$ and $X_{1}^{*}$ denote the closed unit ball of $X$ and of $X^{*}$ respectively, $K \subseteq X$ denotes a convex subset of $X$ and $F: K \multimap K$ will be a proper multimap with convex values. Finally we denote with $\mathcal{B}$ a foundamental system of neighborhoods of the null element 0 in $X$ with respect to the weak topology of $X$, defined by $V=V\left(x_{1}^{*}, \ldots, x_{n}^{*}, \varepsilon\right)=\{x \in X$ : $\left.\left|x_{i}^{*}(x)\right|<\varepsilon, i=1, \ldots, n\right\}$ and $\bar{V}=\left\{x \in X:\left|x_{i}^{*}(x)\right| \leq \varepsilon, i=1, \ldots, n\right\}$.

\section{Properties of fixed point sets}

We begin with a generalization of the piecewise midpoint linearity.

Definition 2.1. Let $K \subseteq X$ and let $F: K \multimap X$ be a multimap with nonempty values. $F$ is said to be strongly nice if there exists $\alpha \geq 1$ such that for every $r>0$ there exists $s(r) \in] 0, r]$ such that

$$
{\overline{F_{s(r)}}}^{w} \subset F_{\alpha s(r)},
$$

where for a set $A, \bar{A}^{w}$ denotes the closure with respect to the weak topology of the space. Analogously we shall say that $F$ is weakly nice provided there exists $\alpha \geq 1$ such that for any $U \in \mathcal{B}$ there exists $V \in \mathcal{B}, V \subseteq U$ such that

$$
{\overline{F_{V}}}^{w} \subset F_{\alpha V} \text {. }
$$

Examples of strongly (resp. weakly)- nice maps are provided by piecewise strongly (resp. weakly) midpoint linear multimaps which are upper semicontinuous from $X$ to $X_{w}$. In fact, as shown in the proof of Theorem 4.3 of [5], for any upper semicontinuous multimap from $X$ to $X_{w}$, the sets $F_{V}$ and $F_{r}$ are closed for any $V \in \mathcal{B}$ and any $r>0$, respectively. Thus if one assumes for instance that $F$ is piecewise weakly midpoint linear, taking any $U \in \mathcal{B}$ and determining the corresponding $V, \mathcal{D}_{V}$ one finds for each $D_{i}$

$$
F_{V} \cap D_{i}=\operatorname{co}\left(F_{V} \cap D_{i}\right) \subset F_{V}
$$

whence

$$
{\overline{F_{V} \cap D_{i}}}^{w}=\overline{F_{V} \cap D_{i}} \subset \overline{F_{V}}=F_{V}
$$

and

$$
{\overline{F_{V}}}^{w}={\overline{\bigcup_{i=1}^{n}\left(F_{V} \cap D_{i}\right)}}^{w} \subset \bigcup_{i=1}^{n}{\overline{F_{V} \cap D_{i}}}^{w} .
$$


The concept of weakly nice multimap is in some sense a generalization of the natural assumption of weak upper semicontinuity. In fact we have the following result

Proposition 2.2. Let $K$ be a convex weakly compact subset of $X, F: K \multimap$ $X$ a weakly upper semicontinuous multimap with nonempty, convex values and suppose $F(K)$ bounded. Then for any $\varepsilon>0$ and, for any $V=V\left(x_{1}^{*}, \ldots, x_{n}^{*}, \alpha\right) \in$ $\mathcal{B}$, it holds

$$
\bar{F}_{V}^{w} \subset F_{(1+\varepsilon+\rho / \alpha) V} .
$$

where $\rho=\sup \{\operatorname{diam} F(x), x \in K\}$.

Proof. Fix $\varepsilon>0$ and $V \in \mathcal{B}$, by the upper semicontinuity of $F$, for any $x \in K$ there exists a neighbourhood, say $U(x) \in \mathcal{B}$, such that

$$
F(z) \subset F(x)+\frac{\varepsilon}{2} V, \quad \text { for any } z \in x+U(x) .
$$

Consider $\left\{x_{1}, x_{2}, \ldots\right\}$ such that $K \subset \bigcup_{i=1}^{n}\left[x_{i}+U\left(x_{i}\right)\right]$ and let $D_{i}=\left[x_{i}+\overline{U\left(x_{i}\right)}\right] \cap K$, $i=1, \ldots, n$. Observe that

$$
\bar{F}_{V}^{w}=\bigcup_{i=1}^{n}\left[\bar{F}_{V}^{w} \cap D_{i}\right] \subset \bigcup_{i=1}^{n} \overline{\operatorname{co}}\left[F_{V} \cap D_{i}\right] .
$$

Fix $i \in\{1,2, \ldots\}$ and $x^{\prime}, x^{\prime \prime} \in D_{i} \cap F_{V}$, then there exists $t^{\prime} \in F\left(x^{\prime}\right)$ and $t^{\prime \prime} \in$ $F\left(x^{\prime \prime}\right)$ such that $x^{\prime}-t^{\prime} \in V$ and $x^{\prime \prime}-t^{\prime \prime} \in V$. By the convexity of $D_{i}$ for any $\lambda \in] 0,1\left[, x_{\lambda}=\lambda x^{\prime}+(1-\lambda) x^{\prime \prime} \in D_{i}\right.$ and hence, by the upper semicontinuity of $F$ we have,

$$
F\left(x_{\lambda}\right) \subset F\left(x_{i}\right)+\frac{\varepsilon}{2} V .
$$

Let $\bar{t} \in F\left(x_{\lambda}\right)$, hence there exists $\tau \in F\left(x_{i}\right)$ and $u \in V$, such that $\bar{t}=\tau+\varepsilon u / 2$. Hence

$$
\begin{aligned}
x_{\lambda}-\bar{t}=\lambda & x^{\prime}+(1-\lambda) x^{\prime \prime}-\bar{t}=\lambda\left(x^{\prime}-t^{\prime}\right)+(1-\lambda)\left(x^{\prime \prime}-t^{\prime \prime}\right) \\
& +\lambda\left(t^{\prime}-\tau^{\prime}\right)+(1-\lambda)\left(t^{\prime \prime}-\tau^{\prime \prime}\right)+\left[\lambda \tau^{\prime}+(1-\lambda) \tau^{\prime \prime}-\tau\right]+(\tau-\bar{t}),
\end{aligned}
$$

where $\tau^{\prime}, \tau^{\prime \prime} \in F\left(x_{i}\right)$, are such that $t^{\prime}=\tau^{\prime}+\varepsilon u^{\prime} / 2, t^{\prime \prime}=\tau^{\prime \prime}+\varepsilon u^{\prime \prime} / 2$, indeed $t^{\prime} \in F\left(x^{\prime}\right) \subset F\left(x_{i}\right)+\varepsilon V / 2$ and $t^{\prime \prime} \in F\left(x^{\prime \prime}\right) \subset F\left(x_{i}\right)+\varepsilon V / 2$. By the convexity of $F\left(x_{i}\right), \lambda \tau^{\prime}+(1-\lambda) \tau^{\prime \prime} \in F\left(x_{i}\right)$, hence

$$
x_{\lambda}-\bar{t} \in \lambda V+(1-\lambda) V+\lambda \frac{\varepsilon}{2} V+(1-\lambda) \frac{\varepsilon}{2} V+\rho X_{1}+\frac{\varepsilon}{2} V=(1+\varepsilon) V+\rho X_{1} .
$$

Since $\alpha X_{1} \subset V$ and $\rho X_{1}=(\rho / \alpha) \alpha X_{1}$ we obtain that

$$
\bigcup_{i=1}^{n} \overline{\mathrm{co}}\left[F_{V} \cap D_{i}\right] \subset F_{(1+\varepsilon+\rho / \alpha) V}
$$

and hence the claimed result. 
REMARK 2.3. Since, for a weakly continuous single valued map $f: K \mapsto X$, with $K \subset X$ convex and weakly compact, $\rho=0$, we have, for any $\varepsilon>0$, that $f$ is $(1+\varepsilon)$-weakly nice.

REMARK 2.4. If the multimap $F$ in Proposition 2.2 has weakly compact values then $F(K)$ is weakly compact, hence, in particular, it is bounded.

On the other side the Example 4.1 of [5] provides an example of a weakly nice multimap which is not weakly upper semicontinuous. The next result justifies the definition of nice multimaps, it provides a structure result for the set of fixed points of a nice multimap.

Given $K \subseteq X$ and a multimap $F: K \multimap X$, with $K \subset X$, we denote with $P(F)$ the set of its fixed points, i.e. $P(F)=\{x \in K: x \in F(x)\}$.

Proposition 2.5. Let $K$ be a subset of $X, F: K \multimap X$ a multimap with nonempty values, which is weakly or strongly nice. Then $P(F)$ is weakly closed.

Proof. Assume $F$ to be weakly nice. It is clear that $P(F)=\bigcap_{V \in \mathcal{B}} F_{V}$. Now $\mathcal{B}=\{\alpha U, U \in \mathcal{B}\}$, then $P(F)=\bigcap_{U \in \mathcal{B}} F_{\alpha U}$ as well. Since, for $V(U) \subset U$, $\alpha V(U) \subset \alpha U$ then $\bigcap_{U \in \mathcal{B}} F_{\alpha V(U)} \subset P(F)$. Hence

$$
\bigcap_{U \in \mathcal{B}}{\overline{F_{V(U)}}}^{w} \subset \bigcap_{U \in \mathcal{B}} F_{\alpha V(U)} \subset P(F) .
$$

On the other side, as $P(F) \subset F_{V(U)} \subset{\overline{F_{V(U)}}}^{w}$, the converse inclusion also holds, and this concludes the proof.

An analogous proof holds if $F$ is assumed to be strongly nice.

REMARK 2.6. Note that nor the weak compactness of $K$ neither the upper semicontinuity of the multimap $F: K \multimap X$ are needed to prove Proposition 2.5.

With the definition of nice multimaps we can generalize the fixed point Theorems 4.2 and 4.3 of [5].

Theorem 2.7. Let $K \subset X$ be convex and weakly compact, and let $F: K \multimap K$ be a weakly nice, upper semicontinuous from $X$ to $X_{w}$ multimap, with nonempty, convex and closed values. Then $P(F)$ is nonempty (and weakly closed).

Proof. Following the same proof as in Theorem 4.3 of [5] one shows that $F_{V}$ is nonempty, without making use of the assumption of midpoint linearity.

Now the family $\left\{F_{V}, V \in \mathcal{B}\right\}$ has the finite intersection property, since when one fixes finitely many weak neighbourhoods $V_{1}, \ldots, V_{n}$, choosing $W=\bigcap_{i=1}^{n} V_{i} \in \mathcal{B}$ one finds $F_{W} \subset F_{V_{i}}, i=1, \ldots, n$, Thus

$$
\emptyset \neq F_{W} \subset \bigcap_{i=1}^{n} F_{V_{i}} .
$$


Consequently, the family $\left\{\overline{F_{V}} w, V \in \mathcal{B}\right\}$ has the finite intersecton property, and from the weak compactness of $K$, there follows $\bigcap_{V \in \mathcal{B}}{\overline{F_{V}}}^{w} \neq \emptyset$, which in turn implies that $\bigcap_{U \in \mathcal{B}} F_{\alpha U} \neq \emptyset$ and this naturally implies that $\bigcap_{U \in \mathcal{B}} F_{U} \neq \emptyset$.

Analogously it is possible to prove the corresponding fixed point theorem for strongly nice multimaps.

Theorem 2.8. Let $K \subset X$ be a convex weakly compact set and $F: K \multimap X$ be a strongly nice, upper semicontinuous and $\gamma$-nonexpansive multimap with closed convex values. Then $P(F)$ is nonempty (and weakly closed).

2.1. Fixed points for a pair of multimaps. We have the following initial result for a pair of multimaps.

Definition 2.9. Two multimaps $F, G: K \subseteq X \multimap X$ commute if $F(G(x))=$ $G(F(x))$ for any $x \in K$.

THEOREM 2.10. Let $K$ be a convex and weakly compact subset of $X, F: K \multimap$ $K$ and $G: K \multimap K$ be two multimaps with convex closed values, satisfying one of the following set of conditions:

(a) $F$ is either u.s.c., $\gamma$-nonexpansive and strongly midpoint linear, or u.s.c. from $X$ to $X_{w}$ and weakly midpoint linear;

(b) $G$ is either u.s.c., $\gamma$-nonexpansive and strongly nice, or u.s.c. from $X$ to $X_{w}$ and weakly nice.

Moreover, we suppose that $F$ and $G$ commute and for any $x \in P(F)$ that $F$ is constant on $G(x)$. Then there exists a common fixed point $x_{0} \in P(F) \cap P(G)$.

Proof. By Theorem 2.8 or 2.7 and by Proposition $2.5 P(F)$ is nonempty and weakly closed, hence weakly compact, as subset of a weakly compact set. Moreover, it holds $G(P(F)) \subset P(F)$. Indeed, for any $x \in P(F)$ and $y \in G(x)$,

$$
y \in G(x) \subseteq G(F(x))=F(G(x))=F(y) .
$$

The last equality is due to the fact that $F$ is constant on $G(P(F))$. Furthermore, by the midpoint linearity of $F, P(F)$ is convex. Therefore if we consider the restriction of the multimap $G$ to the set $P(F)$ we have $G: P(F) \multimap P(F)$ and $G$ satisfies the set of assumptions (b), then applying Theorem 2.8 or 2.7 we obtain the existence of $x_{0} \in G\left(x_{0}\right)$, i.e. $x_{0} \in P(F) \cap P(G)$.

We observe that the requirement $F$ is constant on $G(x)$ for any $x \in P(F)$ is automatically verified if $F$ is a single valued map.

We shall weaken the statement of Theorem 2.10 in two directions: the commutativity assumption and the convexity of the domain $K$.

Using the concept of Banach operator pair introduced by Chen and $\mathrm{Li}$ in [7] for 
single valued maps, it is possible to avoid the requirement $F$ is constant on $G(x)$ for any $x \in P(F)$ and to weaken the commutativity assumption.

Definition 2.11. A pair $(G, F)$ of multimaps, $F, G: X \multimap X$, is a Banach operator pair (BOP) if $G(P(F)) \subseteq P(F)$.

TheOrem 2.12. Let $K$ be a convex and weakly compact subset of $X, F: K \multimap$ $K$ and $G: K \multimap K$ be two multimaps with convex closed values, satisfying one of the following set of conditions:

(a) $F$ is either u.s.c., $\gamma$-nonexpansive and strongly midpoint linear, or u.s.c. from $X$ to $X_{w}$ and weakly midpoint linear;

(b) $G$ is either u.s.c., $\gamma$-nonexpansive and strongly nice, or u.s.c. from $X$ to $X_{w}$ and weakly nice.

Moreover, we suppose that the pair $(G, F)$ is a BOP. Then there exists a common fixed point $x_{0} \in P(F) \cap P(G)$.

Another generalization of the commutativity is the sub-compatibility given by the following definition, which generalizes the so called weak-compatibility introduced by Abbas and Khan in [1] for a pair of a single valued and a multivalued map.

Definition 2.13. A pair $(G, F)$ of two multimpas $F, G: X \multimap X$ is said to be sub-compatible if

$$
G(C(G ; F)) \subseteq C(G, F)
$$

where $C(G, F)$ is the so called coincidence set, i.e.

$$
C(G, F)=\{x \in X: G(x) \cap F(x) \neq \emptyset\} .
$$

We give another fixed point theorem for a pair of sub-compatible multimaps $F, G: X \multimap X$.

ThEOREM 2.14. Let $K$ be a weakly compact subset of $X, F, G: K \multimap X$ be two multimaps with convex closed values, satisfying one of the following set of conditions;

(a) F, $G$ are u.s.c., and $G$ is $\gamma$-nonexpansive and strongly nice; or

(b) $F, G$ are u.s.c. from $X$ to $X_{w}$, and $G$ is weakly nice.

Moreover we suppose that the set $C(G, F)$ is nonempty and convex and that the pair $(G, F)$ is sub-compatible. Then there exists a common fixed point $x_{0} \in$ $P(F) \cap P(G)$.

Proof. The set $C(G, F)$ is closed with respect to the norm topology under assumption (b). Indeed, let $\left(x_{n}\right) \in C(G, F), x_{n} \rightarrow x_{o}$ and suppose by contradiction that $F\left(x_{o}\right) \cap G\left(x_{o}\right)=\emptyset$. Then there exists $x^{*} \in X_{1}^{*}, \varepsilon>0$ such that 
$\left[F\left(x_{o}\right)+V\right] \cap\left[G\left(x_{o}\right)+V\right]=\emptyset$ with $V=V\left(x^{*}, \varepsilon\right)$. By the upper semicontinuity, for $n$ suitably large, we have that $F\left(x_{n}\right) \subset F\left(x_{o}\right)+V, G\left(x_{n}\right) \subset G\left(x_{o}\right)+V$ and therefore $F\left(x_{n}\right) \cap G\left(x_{n}\right)=\emptyset$ thus contradicting the assumption that $x_{n} \in C(G, F)$. Moreover, the set $C(G, F)$ is convex by hypothesis and so it is weakly closed.

Since upper semicontinuity implies upper semicontinuity from $X$ to $X_{w}$, the set $C(G, F)$ is closed also under the set of ussumptions (1).

By the definition of sub-compatibility $C(G, F)$ is $G$-invariant. Therefore we can apply Theorem 2.8 or 2.7 to the restriction of the multimap $G$ to the set $C(G, F)$, obtaining the existence of $x_{0} \in G\left(x_{0}\right) \cap F\left(x_{0}\right)$.

The following result is a trivial sufficient condition to obtain the convexity of $C(G, F)$.

Corollary 2.15. If $G: X \mapsto X$ and $F: X \multimap X$ are convex multimaps, then $C(G, F)$ is convex.

Moreover, in the case of convex multimaps, the condition that $C(F, G)$ is nonempty can be derived by a condition presented by Ky Fan ([12]).

Proposition 2.16. Let $K$ be weakly compact, and let $F, G: K \multimap X$ be convex $X-X_{w}$ upper semicontinuous multimaps with closed and convex values, satisfying the following assumption:

- for each $x \in K$ and each $x^{*} \in X^{*}$ such that $x^{*}(x)=\min _{z \in K} x^{*}(z)$ there exist $u \in F(x)$,

Then $C(F, G) \neq \emptyset$.

Proof. Similarly to the proof of Theorem 3 in [12], consider, for each $x^{*} \in$ $X^{*}$ the sets

$$
\begin{aligned}
& P\left(x^{*}\right)=\left\{x \in K: \max _{u \in F(x)} x^{*}(u) \leq \min _{v \in G(x)} x^{*}(v)\right\}, \\
& Q\left(x^{*}\right)=\left\{x \in K: \max _{u \in F(x)} x^{*}(u)<\min _{v \in G(x)} x^{*}(v)\right\} .
\end{aligned}
$$

Assume, by contradiction, that the assertion is false, namely that $F(x) \cap G(x)=\emptyset$ for each $x \in K$. Then corresponding to each $x \in K$ there exist $x_{x}^{*} \in X^{*}, \alpha_{x} \in \mathbb{R}$ such that $F(x) \subset\left\{z \in X \mid x_{x}^{*}(z)<\alpha_{x}\right\}, G(x) \subset\left\{z \in X \mid x_{x}^{*}(z)>\alpha_{x}\right\}$. Hence $x \in Q\left(x_{x}^{*}\right)$. Since both maps are $X-X_{w}$ upper semicontinuous, the above inclusions keep holding for $z \in x+r X_{1}$ for suitable $r>0$. Hence $Q\left(x_{x}^{*}\right)$ is norm open. Then, for each $x^{*}$, there follows $Q\left(x_{x}^{*}\right) \subset\left[P\left(x_{x}^{*}\right)\right]^{o}$ (where ${ }^{o}$ denotes the norm interior of the set).

Let $x^{*}$ be fixed; we shall prove that $\left[P\left(x^{*}\right)\right]^{c}$ is convex. To this aim let $x_{1}, x_{2} \in\left[P\left(x^{*}\right)\right]^{c}$, i.e. $\min _{v \in G\left(x_{i}\right)} x^{*}(v)<\max _{u \in F\left(x_{i}\right)} x^{*}(u)$. Choose $v_{i} \in G\left(x_{i}\right)$ such that 
$x^{*}\left(v_{i}\right)=\min _{v \in G\left(x_{i}\right)} x^{*}(v)$. Since $G$ is convex, $\lambda v_{1}+(1-\lambda) v_{2} \in G\left(\lambda x_{1}+(1-\lambda) x_{2}\right)$ whence

$\min _{v \in G\left(x_{\lambda}\right)} x^{*}(v) \leq \lambda x^{*}\left(v_{1}\right)+(1-\lambda) x^{*}\left(v_{2}\right)<\lambda \max _{u \in F\left(x_{1}\right)} x^{*}(u)+(1-\lambda) \max _{u \in F\left(x_{2}\right)} x^{*}(u)$.

Again we can choose $u_{i} \in F\left(x_{i}\right)$ that realize the maxima; therefore we have

$$
\min _{v \in G\left(x_{\lambda}\right)} x^{*}(v)<x^{*}\left(\lambda u_{1}+(1-\lambda) u_{2}\right) .
$$

As $u_{i} \in F\left(x_{i}\right)$ and $F$ is convex, $\lambda u_{1}+(1-\lambda) u_{2} \in F\left(x_{\lambda}\right)$ and thus

$$
\min _{v \in G\left(x_{\lambda}\right)} x^{*}(v)<\max _{u \in F\left(x_{\lambda}\right)} x^{*}(u) .
$$

Hence $x_{\lambda} \in\left[P\left(x^{*}\right)\right]^{c}$. Thus the norm closure $\overline{\left[P\left(x^{*}\right)\right]^{c}}$ is convex too, and therefore it is weakly closed. Now

$$
\begin{aligned}
\overline{\left[P\left(x^{*}\right)\right]^{c}}= & {\left[P\left(x^{*}\right)\right]^{c} \cup \partial P\left(x^{*}\right) } \\
= & \left\{x \in X \mid \min _{v \in G(x)} x^{*}(v)<\max _{u \in F(x)} x^{*}(u)\right\} \\
& \cup\left\{x \in X \mid \min _{v \in G(x)} x^{*}(v)=\max _{u \in F(x)} x^{*}(u)\right\}=\left[Q\left(x^{*}\right)\right]^{c}
\end{aligned}
$$

which proves that $Q\left(x^{*}\right)$ is weakly open. Then, clearly $\left\{Q\left(x_{x}^{*}\right), x \in K\right\}$ is a weakly open covering of $K$. Then precisely the same proof as the already mentioned Theorem 3 of Ky Fan ([12]) applies, since $K$ is assumed to be weakly compact.

If the coincidence point set coincides with $K$ we can avoid any hypothesis of commutativity or compatibility.

THEOREM 2.17. Let $K$ be a convex and weakly compact subset of $X, F, G$ : $K \multimap K$ be two multimaps with convex closed values, satisfying one of the following set of conditions:

(a) F, $G$ are u.s.c., strongly nice and $G$ is $\gamma$-nonexpansive;

or

(b) F, $G$ are u.s.c. from $X$ to $X_{w}$ and weakly nice.

Moreover, we suppose that

(c) $F(x) \cap G(x) \neq \emptyset$ for any $x \in K$.

Then there exists a common fixed point $x_{0} \in P(F) \cap P(G)$.

Proof. Define the multimap $H: K \multimap K$ as $H(x)=F(x) \cap G(x)$. We assume the set of conditions (b).

$H$ is upper semicontinuous from $X$ to $X_{w}$ (see [13, p. 52]). Moreover, similarly to the proof of Theorem 4.3 in [5], one shows that $H_{V} \neq \emptyset$ for any 
$V \in \mathcal{B}$ (since the midpoint linearity is of no use for this step). Therefore, since $H_{V} \subseteq F_{V} \cap G_{V}$, we obtain $F_{V} \cap G_{V} \neq \emptyset$ for any $V \in \mathcal{B}$.

Let now $\alpha \geq 1$ be such that ${\overline{F_{V}}}^{w} \subset F_{\alpha V}$ and $\bar{G}_{V}{ }^{w} \subset G_{\alpha V}$. Note that

$$
F_{V} \cap G_{V} \subset{\overline{F_{V}}}^{w} \cap{\overline{G_{V}}}^{w} \subset{\overline{F_{V}}}^{w} \subset F_{\alpha V},
$$

analogously $F_{V} \cap G_{V} \subset G_{\alpha V}$. Hence $\left\{{\overline{F_{V}}}^{w} \cap{\overline{G_{V}}}^{w}, V \in \mathcal{B}\right\}$ has the finite intersection property, since the family $\left\{F_{V} \cap G_{V}, V \in \mathcal{B}\right\}$ satisfies it. Thus, from the weak compactness of $K$ we have

$$
\bigcap_{V \in \mathcal{B}}\left({\overline{F_{V}}}^{w} \cap{\overline{G_{V}}}^{w}\right) \neq \emptyset,
$$

yielding in turn that

$$
\bigcap_{V \in \mathcal{B}}\left(F_{\alpha V} \cap G_{\alpha V}\right) \neq \emptyset
$$

and thus

$$
\bigcap_{U \in \mathcal{B}}\left(F_{U} \cap G_{U}\right) \neq \emptyset
$$

hence obtaining the existence of a common fixed point in the case of the set of hypotheses (b).

In the case of the set of hypotheses (a), it is easy to prove that the multimap $H$ is $\gamma$-nonexpansive. Hence, following the proof of Theorem 4.2 in [5], it is possible to prove that $H_{r} \neq \emptyset$ for any $r>0$. Then, with the same argument of the proof above, one reaches $\bigcap_{r>0}\left(F_{r} \cap G_{r}\right) \neq \emptyset$.

Note that Theorems 2.10 and 2.12 differ from Theorems 2.14 and 2.17 in the topological indipendence of the assumptions: more precisely, in Theorems 2.10 and 2.12 the topologies (strong or weak) with respect to which the assumptions on $F$ and $G$ are formulated may be different, while in Theorems 2.14 and 2.17 the semicontinuity should be for both with respect to the same topology.

If in Theorem 2.12 we assume that $F$ and $G$ are nonexpansive, then we can replace the assumption of convexity of $K$ with its starshapedness: furthermore, if $F$ is contractive then we just need that $K$ is weakly compact.

First of all we show a fixed point theorem for a multivalued map with a starshaped domain.

THEOREM 2.18. Let $K$ be a starshaped weakly compact subset of $X$ and let $F: K \multimap K$ be a nonexpansive strongly nice multimap with closed values, then there exists a fixed point $x_{0} \in F\left(x_{0}\right)$.

Proof. Let $p \in X$ be the center of starshapedness of $K$. For any $\lambda \in] 0,1[$ consider the following multimap

$$
F_{(\lambda)}(x)=\lambda F(x)+(1-\lambda) p .
$$


Since $K$ is starshaped, $F_{(\lambda)}$ maps $K$ into itself. Moreover since $F$ is nonexpansive, for any $x, y \in K$, we have

$$
d_{H}\left(F_{(\lambda)}(x), F_{(\lambda)}(y)\right)=d_{H}(\lambda F(x), \lambda F(y))=\lambda d_{H}(F(x), F(y)) \leq \lambda\|x-y\|,
$$

i.e. $F_{(\lambda)}$ is contractive. Now we can apply Theorem 11.1 in [9] obtaining that $P\left(F_{(\lambda)}\right) \neq \emptyset$. Following the proof of Theorem 4.2 of [5] it is possible to show that $F_{r} \neq \emptyset$ for any $r>0$; then by Theorem 2.8 the assertion follows.

Now we prove an analogous theorem for a pair of multimaps.

THEOREM 2.19. Let $K$ be a starshaped weakly compact subset of $X$ and let $F: K \multimap K$ and $G: K \multimap K$ be two nonexpansive strongly nice multimaps with closed values. Moreover, we suppose that $P(F)$ is starshaped and that the pair $(G, F)$ is a BOP. Then there exists a common fixed point $x_{0} \in P(F) \cap P(G)$.

Proof. By Theorem 2.18 we have that $P(F) \neq \emptyset$ and by Proposition 2.5 we obtain that the set $P(F)$ is weakly closed, hence weakly compact as a subset of $K$. Moreover, by the BOP definition, $P(F)$ is invariant under $G$. Therefore, applying again Theorem 2.18 to the restriction of the multimap $G$ to the set $P(F)$, we obtain the existence of $x_{0} \in G\left(x_{0}\right)$, i.e. $x_{0} \in P(F) \cap P(G)$.

REMARK 2.20. If in Theorem 2.19 we assume that $G$ is contractive, no assumption on $P(F)$ is needed.

REMARK 2.21. In complete analogy, one can replace the assumption of convexity of $C(G, F)$ in Theorem 2.14 with the assumption that $C(G, F)$ is starshaped and $G$ is nonexpansive. Furthermore, again when $G$ is contractive no assumption on $C(G, F)$ is needed besides $C(G, F) \neq \emptyset$.

2.2. Fixed points for families of multimaps. Throughout this section $I$ will denote a nonempty set of indexes and $F_{\eta}$ a multimap $F_{\eta}: K \multimap K$.

The following corollary is an immediate consequence of Proposition 2.5.

Corollary 2.22. Let $K \subset X$ be a weakly compact set and $\mathcal{F}=\left\{F_{\eta}, \eta \in I\right\}$ be a family of multimaps, each with nonempty values and such that each $F_{\eta}$ is either strongly or weakly nice. If $\mathcal{F}$ satisfies the condition:

(a) $\{P(F), F \in \mathcal{F}\}$ has the finite intersection property, then $\mathcal{F}$ has a common fixed point.

We shall now show some conditions that imply condition (a) of Corollary 2.22 . The first one is inspired by [16].

Definition 2.23. A family of multimaps $\mathcal{F}$ from $K$ to $X$ is said to be commutative if for any $F, G \in \mathcal{F}$ it holds $F(G(x))=G(F(x))$ for any $x \in K$. 
TheOREM 2.24. Let $K \subset X$ be a convex weakly compact set and $\mathcal{F}=\left\{F_{\eta}, \eta \in\right.$ $I\}$ be a commutative family of multimaps, each with convex closed values and such that each $F_{\eta}$ is either

(a) u.s.c., $\gamma$-nonexpansive and strongly midpoint linear,

or

(b) u.s.c. from $X$ to $X_{w}$ and weakly midpoint linear.

If for any $F_{\eta}, F_{\vartheta} \in \mathcal{F}$ and for any $x \in P\left(F_{\eta}\right), F_{\eta}$ is constant on $F_{\vartheta}(x)$, then $\mathcal{F}$ satisfies condition $(\beta)$, and therefore it has a common fixed point.

Proof. The set $\left\{P\left(F_{\eta}\right), \eta \in I\right\}$ has the finite intersection property. Indeed, let $F_{\eta}, F_{\vartheta} \in \mathcal{F}$, with the same argument as in Theorem 2.10 it is possible to obtain the existence of $x_{0} \in P\left(F_{\eta}\right) \cap P\left(F_{\vartheta}\right)$.

We can repeat this reasoning for any finite set $\left\{F_{\eta_{1}}, \ldots, F_{\eta_{n}}\right\} \subset \mathcal{F}$. Hence the set $\left\{P\left(F_{\eta}\right), \eta \in I\right\}$ has the finite intersection property. In conclusion by Corollary 2.22 we obtain the claimed result.

Observe that for this result we have to assume the (global) midpoint linearity instead of the weaker assumption of strong or weak nicety, since this last requirement does not ensure the convexity of each $P\left(F_{\eta}\right)$ where we want to apply a Fixed Point Theorem.

A corollary of Theorem 2.24 is the following.

Corollary 2.25. Let $K \subset X$ be a convex weakly compact set and $\mathcal{F}=$ $\left\{F_{\eta}, \eta \in I\right\}$ be a commutative family of convex, $X-X_{w}$ upper semicontinuous multimaps such that $F_{\eta}(x) \subset K$ is a closed set for any $x \in K$ and every $F_{\eta} \in \mathcal{F}$. We assume for each pair $F_{\eta}, F_{\vartheta} \in \mathcal{F}$ and each fixed point $x \in P\left(F_{\eta}\right), F_{\eta}$ is constant on $F_{\vartheta}(x)$. Then $\mathcal{F}$ has a common fixed point.

The previous corollary is a generalization of Theorem 1 of [16], where a common fixed point is proved for a family of weakly continuous multimaps.

In analogy with Theorem 2.24, it is possible to extend Theorem 2.17 to a family of multimaps, proving that $\left\{P\left(F_{\eta}\right), \eta \in I\right\}$ is a family of weakly closed sets with the finite intersection property.

THEOREM 2.26. Let $K \subset X$ be a weakly compact set and $\mathcal{F}=\left\{F_{\eta}, \eta \in I\right\}$ be a family of multimaps, each with convex closed values and satisfying one of the following set of conditions:

(a) $F_{\eta}$ is u.s.c. and strongly nice, with at least one $F_{\eta} \in \mathcal{F} \gamma$-nonexpansive; or

(b) $F_{\eta}$ is u.s.c. from $X$ to $X_{w}$ and weakly nice.

Moreover, we suppose that 
(c) for any $J=\left\{\eta_{1}, \ldots, \eta_{n}\right\} \subseteq I$ there holds $\bigcap_{i=1}^{n} F_{\eta_{i}}(x) \neq \emptyset$ for any $x \in K$.

Then there exists a common fixed point for $\mathcal{F}$.

In [3] the following common fixed point theorem is proven.

Theorem 2.27 ([3, Theorem 1]). Let $K$ be a nonempty convex subset of a topological vector space, $\mathcal{F}=\left\{F_{\eta}, \eta \in I\right\}$, be a family of multimaps satisfying the following conditions:

(a) for each $\eta \in I$, the set $P\left(F_{\eta}\right)$ is closed;

(b) $\bigcup_{\eta \in I} F_{\eta}$ is a compact multimap;

(c) for each finite set $\left\{\eta_{1}, \ldots, \eta_{k}\right\} \subset I$ there exists $\left\{z_{1}, \ldots, z_{k}\right\} \subset K$ such that

$$
\operatorname{co}\left\{z_{i}, i \in J\right\} \subset \bigcup_{i \in J} F_{\eta_{i}}(x),
$$

for each $x \in K$, and for each subset $J \subset\{1, \ldots, n\}$.

Then there exists $x_{0} \in \bigcap_{\eta \in I} P\left(F_{\eta}\right)$.

Here we state the following similar result without the assumption of compactness of the family of multimaps.

TheOREM 2.28. Let $K$ be a nonempty convex subset of a topological vector space, $\mathcal{F}=\left\{F_{\eta}, \eta \in I\right\}$ be a family of multimaps satisfying the following conditions:

(a) for each $\eta \in I$, the set $P\left(F_{\eta}\right)$ is closed;

(b) there exists $\eta_{o}$ such that $P\left(F_{\eta_{o}}\right)$ is compact;

(c) for each finite set $\left\{\eta_{1}, \ldots, \eta_{k}\right\} \subset I$ there exists $\left\{z_{1}, \ldots, z_{k}\right\} \subset K$ such that

$$
\operatorname{co}\left\{z_{i}, i \in J\right\} \subset \bigcup_{i \in J} F_{\eta_{i}}(x),
$$

for each $x \in K$, and for each subset $J \subset\{1, \ldots, n\}$.

Then the family of multimaps has a common fixed point.

Proof. First note that for each $\eta \in I$ the set $P\left(F_{\eta}\right)$ is nonempty; in fact, fix $\eta \in I$ and apply (3); then there exists $z \in K$ such that $\{z\} \subset F_{\eta}(x)$ for every $x \in K$. In particular $z \in F_{\eta}(z)$ and hence $z \in P\left(F_{\eta}\right)$.

We prove that $\left\{P\left(F_{\eta}\right), \eta \in I\right\}$ has nonempty intersection. Suppose conversely that $\bigcap_{\eta \in I} P\left(F_{\eta}\right)=\emptyset$, hence $\bigcap_{\eta \in I, \eta \neq \eta_{o}} P\left(F_{\eta}\right) \cap P\left(F_{\eta_{o}}\right)=\emptyset$ Then by assumption (a) and the compactness of $P\left(F_{\eta_{o}}\right)$ there should exist $\eta_{1}, \ldots, \eta_{k} \in I$ such that $\bigcap_{i=1}^{k} P\left(F_{\eta_{i}}\right)=\emptyset$ and thus $K=\bigcup_{i=1}^{k} P\left(F_{\eta_{i}}\right)^{c}$. The remaining of the proof is the same as in the mentioned Balaj's result. 
The weakly nice condition gives a sufficient condition for the closure of the fixed point set. More precisely we have the following corollary.

COROllary 2.29. Let $K$ be a nonempty convex weakly compact subset of $X$, $\mathcal{F}=\left\{F_{\eta}, \eta \in I\right\}$ be a family of multimaps satisfying the following conditions:

(a) for each $\eta \in I$, the multimap $F_{\eta}$ is weakly nice;

(b) there exists $\eta_{o}$ such that $P\left(F_{\eta_{o}}\right)$ is weakly compact;

(c) for each finite set $\left\{\eta_{1}, \ldots, \eta_{k}\right\} \subset I$ there exists $\left\{z_{1}, \ldots, z_{k}\right\} \subset K$ such that

$$
\operatorname{co}\left\{z_{i}, i \in J\right\} \subset \bigcup_{i \in J} F_{\eta_{i}}(x),
$$

for each $x \in K$, and for each subset $J \subset\{1, \ldots, n\}$.

Then the family of multimaps has a common fixed point.

\section{Applications}

3.1. Best approximations. Let $C$ be a subset of a Banach space $X$. For $x_{0} \in X$, we denote by $P_{C}\left(x_{0}\right)$ the set of best $C$-approximants to $x_{0}$, i.e.

$$
P_{C}\left(x_{0}\right)=\left\{y \in C:\left\|y-x_{0}\right\|=\inf _{z \in C}\left\|z-x_{0}\right\|\right\} .
$$

We recall that if $P_{C}(x) \neq \emptyset$ for any $x \in X$ the set $C$ is called proximinal. An easy example of a proximinal set is a closed convex subset of a Hilbert space. It is well known that $P_{C}\left(x_{0}\right)$ is bounded and is closed and convex if $C$ is closed and convex, for all these results see [22, (p. 73-76)]. Moreover, the following results are straightforward.

REMARK 3.1. If $C$ is weakly compact then $P_{C}\left(x_{0}\right)$ is weakly compact. If $X$ is a reflexive Banach space then $P_{C}\left(x_{0}\right)$ is weakly compact.

The following result is an immediate consequence of the common fixed point theorems proved in the previous section.

Proposition 3.2. Let $C$ be a nonempty subset of $K$, where $K$ is a convex weakly compact subset of $X$. Let $K$ be a convex and weakly compact subset of $X, F: K \multimap K$ and $G: K \multimap K$ be two multimaps with convex closed values, satisfying one of the following set of conditions.

(a) $F$ is either u.s.c., $\gamma$-nonexpansive and strongly midpoint linear, or u.s.c. from $X$ to $X_{w}$ and weakly midpoint linear;

(b) $G$ is either u.s.c., $\gamma$-nonexpansive and strongly nice, or u.s.c. from $X$ to $X_{w}$ and weakly nice.

Moreover, we suppose that the pair $(G, F)$ is a BOP. Let $x_{0} \in P(F) \cap P(G)$, the set $P_{C}\left(x_{0}\right)$ be nonempty weakly compact and convex and $P_{C}\left(x_{0}\right)$ be invariant under $F$ and $G$. Then $P_{C}\left(x_{0}\right) \cap P(F) \cap P(G) \neq \emptyset$. 
If in Theorem 3.2 we assume that $F$ and $G$ are nonexpansive, then $P_{C}\left(x_{0}\right)$ is invariant under $F$ and $G$ and we can replace the assumption of convexity of $P_{C}\left(x_{0}\right)$, with its starshapedness: furthermore if $F$ and $G$ are contractive, then we just need $P_{C}\left(x_{0}\right)$ to be nonempty and weakly compact.

Proposition 3.3. Let $C$ be a nonempty subset of $K$, where $K$ is a weakly compact starshaped subset of $X$. Let $F: K \multimap K$ and $G: K \multimap K$ be nonexpansive with closed values such that $F$ is strongly midpoint linear and $G$ is strongly nice. Moreover we suppose that the pair $(G, F)$ is a BOP. Let $x_{0} \in P(F) \cap P(G)$ and the set $P_{C}\left(x_{0}\right)$ be nonempty, weakly compact and starshaped. Then $P_{C}\left(x_{0}\right) \cap P(F) \cap P(G) \neq \emptyset$.

Proof. We have that $P_{C}\left(x_{0}\right)$ is invariant under $F$ and $G$. Indeed by hypothesis there exists a constant $k \in] 0,1\left[\right.$ such that, for any $x \in P_{C}\left(x_{0}\right)$ and $y \in F(x)$,

$$
\left\|y-x_{0}\right\| \leq d_{H}\left(F(x), F\left(x_{0}\right)\right) \leq k\left\|x-x_{0}\right\|<\left\|x-x_{0}\right\|,
$$

thus $y \in P_{C}\left(x_{0}\right)$. The same is true for $G$.

Now, recalling that if $G$ is nonexpansive, then it is $\gamma$-nonexpansive, with respect to a measure of noncompactness $\gamma$, we can apply Theorem 2.19 to the restriction of $F$ and $G$ to $P_{C}\left(x_{0}\right)$ obtaining a common fixed point in $P_{C}\left(x_{0}\right)$.

We show now a condition that imply the starshapeness of $P_{C}\left(x_{0}\right)$.

Proposition 3.4. Let $C \subset X$ be starshaped and, denoting with

$$
S(C)=\{y \in C: C \text { is starshaped with respect to } y\},
$$

assume $S(C) \cap P_{C}\left(x_{0}\right) \neq \emptyset$ then $P_{C}\left(x_{0}\right)$ is starshaped with respect to $p \in S(C) \cap$ $P_{C}\left(x_{0}\right)$.

3.2. Minimax and variational inequalities. From Theorem 2.28 we deduce a minimax inequality without compactness.

Proposition 3.5. Let $K$ be a nonempty convex subset of a topological vector space $X$. Let $f, g: K \times K \rightarrow \mathbb{R}$, be two functions satisfying the following conditions:

(a) for any $x \in K$ there exists $z \in K$ such that $f(x, y) \leq g(z, y)$ for any $y \in Y$;

(b) $f$ is lower semicontinuous in the second variable and $g$ is upper semicontinuous on $\Delta_{x}$;

(c) $g$ is quasi concave in the first variable;

(d) there exists $\bar{x} \in K$ such that the set $\{y \in Y: f(\bar{x}, y)-g(y, y) \leq 0\}$ is compact.

Then there exists $y_{o} \in Y$ such that $f\left(x, y_{0}\right) \leq g\left(y_{o}, y_{o}\right)$ for any $x \in K$. 
Proof. Let $x \in K$, and denote with $F_{x}: K \multimap K$ the multimap defined as

$$
F_{x}(y)=\{z \in K: f(x, y) \leq g(z, y)\} .
$$

Following the proof of Theorem 4 of [3] it is possible to prove that the family of multimaps $\left\{F_{x}, x \in K\right\}$ satisfies conditions (a) and (c) of Theorem 2.28 while condition $(\mathrm{d})$ is equivalent to assuming $P\left(F_{\bar{x}}\right)$ compact. Hence all the hypotheses of Theorem 2.28 are satisfied and so we obtain a common fixed point for the family $\left\{F_{x}, x \in K\right\}$, i.e. the claimed result.

For the particular case of $X$ being a reflexive Banach space we have the following result.

Proposition 3.6. Let $K$ be a nonempty convex subset of a reflexive $B a$ nach space $X$. Let $f, g: K \times K \rightarrow \mathbb{R}$, be two functions satisfying the following conditions:

(a) for any $x \in K$ there exists $z \in K$ such that $f(x, y) \leq g(z, y)$ for any $y \in Y$;

(b) $f$ is weakly lower semicontinuous in the second variable and $g$ is weakly upper semicontinuous on $\Delta_{x}$;

(c) $g$ is quasi concave in the first variable;

(d) there exists $\bar{x} \in K$ such that $\liminf _{\|y\| \rightarrow \infty}(f(\bar{x}, y)-g(y, y))>0$.

Then there exists $y_{o} \in Y$ such that $f\left(x, y_{o}\right) \leq g\left(y_{o}, y_{o}\right)$ for any $x \in K$.

Proof. Condition (d) implies the boundedness of the set $\{y \in K: f(\bar{x}, y)-$ $g(y, y) \leq 0\}$. By conditions (b) it is possible to prove that it is weakly closed and by the reflexivity of the space $X$ we obtain that it is weakly compact, the rest of the proof is analogous to the one of Proposition 3.5.

We can also apply Theorem 2.26 to deduce a variational inequality similar to those appearing in [19] Theorem XV and in [20] Theorem XV.

Proposition 3.7. Let $K \subset X$ be weakly compact and convex and let $p: K \times$ $K \rightarrow \mathbb{R}, h: K \rightarrow \mathbb{R}$ be two functions satisfying:

(a) for any $(x, y) \in K \times K$ there exists $z \in K$ such that $p(x, y) \leq h(x)-h(z)$;

(b) $h$ is lower semicontinuous and quasi-convex;

(c) $y \mapsto p(x, y)$ is weakly lower semicontinuous.

Then there exists $y_{0} \in K$ such that

$$
p\left(x, y_{o}\right)+h\left(y_{o}\right) \leq h(x) \quad \text { for any } x \in K .
$$

Proof. For any $x \in K$ let $F_{x}: K \multimap K$ the multimap defined as

$$
F_{x}(y)=\{z \in K: p(x, y) \leq h(x)-h(z)\} .
$$


By conditions (a) and (b), $F_{x}(y)$ is a nonempty closed and convex set for any $(x, y) \in K \times K$. Next we show that the multimap $F_{x}$ is weakly upper semicontinuous.

Let $y_{n} \rightarrow y_{o}$ and $z_{n} \in F_{x}\left(y_{n}\right)$ for any $n \in \mathbb{N}$ such that $z_{n} \rightarrow z_{o}$. Then, since $h$ and $p(x, \cdot)$ are weakly lower semicontinuous we have

$$
h\left(z_{o}\right)+p\left(x, y_{o}\right) \leq \liminf _{n \rightarrow \infty} h\left(z_{n}\right)+p\left(x, y_{n}\right) \leq h(x) \quad \text { for any } x \in K .
$$

Hence $z_{o} \in F_{x}\left(y_{o}\right)$,obtaining that for any $x \in K, F_{x}$ is a weakly closed multimap. Then by the weak compactness of $K, F_{x}$ is weakly upper semicontinuous. By Proposition 2.2, then each $F_{x}, x \in K$ is weakly nice.

Finally, fix $\left\{x_{1}, \ldots, x_{n}\right\} \subset K, y \in K$. Let $i(y) \in\{1, \ldots, n\}$ be such that

$$
h\left(x_{i(y)}\right)-p\left(x_{i(y)}, y\right)=\min _{1 \leq i \leq n}\left[h\left(x_{i}\right)-p\left(x_{i}, y\right)\right] .
$$

Then $F_{x_{i(y)}}(y) \subset F_{x_{i}}(y), i=1, \ldots, n$ and hence $\bigcap_{i=1}^{n} F_{x_{i}}(y) \neq \emptyset$. In conclusion we can apply Theorem 2.26 obtaining a common fixed point for the family $\left\{F_{x}\right\}_{x \in K}$, i.e. the claimed result.

Note that, the above result cannot be deduced from Theorem XV of [19] (for the case of $q=0, K$ convex and weakly compact in a Banach space). In fact although conditions (b) and (c) do imply assumptions (15.2) and (15.3) there, our first condition is weaker then (15.1), namely $p(x, y) \leq 0$ for any $(x, y) \in K \times K$, as the following example shows.

ExAmple 3.8. Let $K$ be a weakly compact convex subset of $X_{+}$, the cone of a Banach lattice $X$, with $0 \in K$, and let $x^{*} \in\left(X_{1}^{*}\right)_{+}$be fixed. Define

$$
p(x, y)=\min \left\{x^{*}(x), x^{*}(y)\right\}, \quad h(x)=\|x\| .
$$

Then

(a) for any $(x, y) \in K \times K$, we have that $p(x, y) \leq x^{*}(x) \leq\|x\|$, i.e. condition (a) of Theorem 3.7 holds with $z=0$;

(b) $h$ is continuous and convex;

(c) $y \mapsto p(x, y)$ is weakly lower semicontinuous.

Hence there exists $y_{o} \in K$ such that $p\left(x, y_{o}\right)+\left\|y_{o}\right\| \leq\|x\|$ for any $x \in K$.

However, since $p(x, y) \geq 0$ for any $(x, y) \in K \times K$, this result cannot be deduced from Theorem XV of [19].

In the same framework, we can deduce further variational inequalities of Park's type without compactness from Proposition 3.5. More precisely we have the following statement.

Proposition 3.9. Let $K$ be a convex set of a topological vector space $X$ and $u, v: K \times K \rightarrow \mathbb{R}$ such that 
(a) for any $x \in K$ there exists $z \in K$ such that $u(y, y)-u(x, y) \leq v(y, y)-$ $v(z, y)$

(b) the map $y \mapsto u(y, y)-u(x, y)$ is lower semicontinuous;

(c) $v$ is quasi convex in the first variable;

(d) there exists $\bar{x}$ such that the set $\{y \in K: u(y, y)-u(\bar{x}, y) \leq 0\}$ is compact.

Then there exists $y_{o} \in K$ such that $u\left(x, y_{o}\right) \geq u\left(y_{o}, y_{o}\right)$ for any $x \in K$.

Proof. We apply Proposition 3.5 to the functions $f, g: K \times K \rightarrow \mathbb{R}$ defined as

$$
f(x, y)=u(y, y)-u(x, y), \quad g(x, y)=v(y, y)-v(x, y) .
$$

Remark 3.10. Condition (a) of Proposition 3.9 can be obtained if $u \leq v$ on $\Delta$ and for any $x \in K$, there exists $z \in K$ such that $u(x, y) \geq v(z, y)$, for all $y \in K$. Park [19], [20] requires that $u \leq v$ on $\Delta$ and $v \leq u$ on $K \times K \backslash \Delta$, which therefore implies condition (a) above.

Remark 3.11. If $X$ is a reflexive Banach space condition (d) in Proposition 3.9 is fulfilled if there exists $\bar{x} \in K$ such that

$$
\lim _{\|y\| \rightarrow \infty}[u(y, y)-u(\bar{x}, y)]>0 .
$$

\section{REFERENCES}

[1] M. AbBas And A.R. Khan, Common fixed points of generalized contractive hybrid pairs in symmetric spaces, Fixed Point Theory and Appl. (2009), 1-11.

[2] J.P. Aubin And A. Cellina, Differential Inclusions. Set Valued Maps and Viability Theory, Fundamental Principle of Mathematical Sciences, 264, Springer Verlag, 1984.

[3] M. BALAJ, A common fixed point theorem with applications to vector equilibrium problems, Appl. Math. Lett. 23 (2010), 241-245.

[4] J. Banas and K. Goebel, Measure of noncompactness in Banach spaces, Lecture Notes in Pure and Applied Mathematics, 1980.

[5] I. Benedetti, S. Bolognini and A. Martellotti, Multivalued fixed point theorems without strong compactness via a generalization of midpoint convexity, Fixed Point Theory 15 (2014), no. 1, 3-22.

[6] T. Cardinali and P. Rubbioni, Multivalued fixed point theorems in terms of weak topology and of measure of weak noncompactness, J. Math. Anal. Appl. 405 (2013), 409-415.

[7] J. Chen AND Z. LI, Common fixed points for Banach operator pairs in best approximation, J. Math. Anal. Appl. 336 (2007), 1466-1475.

[8] M. Chermisi And A. Martellotti, Fixed point theorems for middle point linear operators in $L^{1}$, Fixed Point Theory Appl. 2 (2008), 103-112.

[9] K. Deimling, Multivalued Differential Equations, Walter de Gruiter, New York, 1992.

[10] G. Emmanuele, Measure of weak noncompactness and fixed point theorems, Bull. Math. Soc. Sci. Math. R.S. Roumanie (N.S.) 25 (1981), 253-258.

[11] K. FAn, A generalization of Tychonoff's fixed point theorem, Math. Ann. 142 (1961), $305-310$ 
[12] _ A Minimax Inequality and Applications, Inequalities, III (Proc. Third Sympos., Univ. California, Los Angeles, Calif., 1969; dedicated to the memory of Theodore S. Motzkin), (1972), 103-113.

[13] S. Hu and N. Papageorgiou, Handbook of Multivalued Analysis, Kluwer Academic Publisher, London, 1997.

[14] L.V. Kantorovich And G.P. Akilov, Functional Analysis, Pergamon Press, Oxford, 1982.

[15] A.R. Khan, N. Hussain And A.B. Thaheem, Applications of fixed point theorems to invariant approximations, Approx. Theory Appl. 16, no. 3 (2000), 48-55.

[16] W.K. KIM AND D.I. RiM, A generalization of the Markov-Kakutani fixed point theorem, J. Chungcheong Math. Soc. 14 (2001), 105-111.

[17] K. Nikodem, On midpoint convex set-valued functions, Aequationes Math. 33 (1987), 46-56.

[18] D. O'Reagan, Fixed point theory of Monch type for weakly sequentially upper semicontinuous maps, Bull. Austral. Math. Soc. 61 (2000), 439-449.

[19] S. PARK, The KKM principle in abstract convex spaces: equivalent formulations and applications, Nonlinear Anal. 73 (2010), 1028-1042.

[20] - New generalizations of basic theorems in KKM theory, Nonlinear Anal. 74 (2011), 3000-3010.

[21] H.K. Pathak, Y.J. Cho And S.M. Kang, An application of fixed point theorems in best approximation theory, Int. J. Math. Math. Sci. 21, no. 3 (1998), 467-470.

[22] S. Singh, B. Watson and P. Srivastava, Fixed Point Theory and Best Approximations: the KKM-Map Principle, Kluwer Academic Publisher, London, 1997.

Manuscript received January 16, 2013

Irene Benedetti And Anna Martellotti

Departmentt of Mathematics and Computer Science

University of Perugia

I-06123 Perugia, ITALY

E-mail address: irene.benedetti@unipg.it, anna.martellotti@unipg.it 